\title{
Eucalyptone G, a new phloroglucinol derivative and other constituents from Eucalyptus globulus Labill
}

\author{
Gamal A. Mohamed ${ }^{\mathrm{a} *}$ and Sabrin R. M. Ibrahim ${ }^{\mathrm{b}}$ \\ ${ }^{a}$ Pharmacognosy Department, Faculty of Pharmacy, Al-Azhar University, Assiut branch, \\ Assiut 71524, Egypt \\ ${ }^{b}$ Pharmacognosy Department, Faculty of Pharmacy, Assiut University, Assiut 71526, Egypt \\ E-mail: gamals2001@yahoo.com
}

\begin{abstract}
A new pholoroglucinol derivative named eucalyptone $G$ (4) together with nine known compounds (1-3, 5-10) were isolated for the first time from the ethyl acetate fraction of the bark of small twigs of Eucalyptus globulus Labill. Their chemical structures were established on the basis of physical, chemical and spectroscopic methods $1 \mathrm{D}\left({ }^{1} \mathrm{H}\right.$ and $\left.{ }^{13} \mathrm{C}\right) \mathrm{NMR}$ and $2 \mathrm{D}(\mathrm{COSY}$, HMQC and HMBC) NMR in addition to mass spectrometry and comparison with literature data. Eucalyptone G displayed antibacterial activity against Bacillus subtilis, Staphylococcus aureus and Escherichia coli.
\end{abstract}

Keywords: Eucalyptus globulus Labill.; Myrtaceae; eucalyptone G, antibacterial activity

\section{Introduction}

Eucalyptus L'He'ritier (Myrtaceae) is one of the world's most important and most widely planted genera. In Australia, this genus is the second largest genus, after Acacia, and contains about 750 species. ${ }^{1}$ Myrtaceous plants are known to be rich source of biologically active terpenoids and polyphenols, including flavonoids, phloroglucinol derivatives, and tannins ${ }^{2}$. Previous phytochemical studies on the Eucalyptus globulus Labill. reported the isolation of sesquiterpenes aromadendrene ${ }^{1}$, acetogenin-sesquiterpenes, acetogenin monoterpenes ${ }^{3-6}$, phloroglucinol sesquiterpenes ${ }^{7}$, and $C$-methyl flavones ${ }^{8}$. The phloroglucinol compounds exhibit interesting biological activities ${ }^{9}$. Eucalyptus globulus Labill. (Myrtaceae) is used as a traditional remedy in many parts of the world for treatment of a wide variety of diseases including microbial infections ${ }^{5}$. In the present work we report the isolation and structure elucidation of one new phloroglucinol derivative named eucalyptone $G$, together with nine known compounds. The antibacterial activity of the new compound has been studied. 


\section{Results and Discussion}

The methanolic extract of the powdered bark of Eucalyptus globulus Labill. was concentrated to dryness and successively partitioned between $n$-hexane, ethyl acetate, $n$-butanol and water. The ethyl acetate fraction afforded one new phloroglucinol derivative named eucalyptone G (4) and nine known compounds (1-3, 5-10).

Rhodomyrtone (3) appeared as orange-coloured spot on silica gel TLC plates when sprayed with anisaldehyde $/ \mathrm{H}_{2} \mathrm{SO}_{4}$ reagent. The ESI-MS spectrum showed pseudomolecular ion peaks at $m / z 443(100)[\mathrm{M}+\mathrm{H}]^{+}$and $907[2 \mathrm{M}+\mathrm{Na}]^{+}$. The UV spectrum exhibited three characteristic bands at $\lambda_{\max }(\mathrm{MeOH}) 224.3,263.1$ and $301.3 \mathrm{~nm} .{ }^{1} \mathrm{H}$ NMR spectrum revealed the presence of three downfield signals; two for $\mathrm{OH}$ groups at $\delta_{\mathrm{H}} 13.04,7.59$ (each $1 \mathrm{H}, \mathrm{s}$ ), and one aromatic proton at $\delta_{\mathrm{H}} 6.11(1 \mathrm{H}, \mathrm{s}, \mathrm{H}-5)$, in addition to methine proton at $\delta_{\mathrm{H}} 4.26(1 \mathrm{H}, \mathrm{t}, J=5.4 \mathrm{~Hz}, \mathrm{H}-9)$. Furthermore, the spectrum exhibited characteristic signals for isovaleryl group at $\delta_{\mathrm{H}} 2.99(2 \mathrm{H}$, $\left.\mathrm{dd}, J=6.8,15.5 \mathrm{~Hz}, \mathrm{H}-2^{\prime}\right), 2.28\left(1 \mathrm{H}, \mathrm{m}, \mathrm{H}-3^{\prime}\right)$, and $0.98(6 \mathrm{H}, \mathrm{d}, J=6.6 \mathrm{~Hz}$, two methyls at C$\left.3^{3}\right)$. On other hand, the ${ }^{1} \mathrm{H}$ NMR revealed the presence of isobutyl group at $\delta_{\mathrm{H}} 1.48(2 \mathrm{H}, \mathrm{m}, \mathrm{H}-$ $1^{\prime \prime)}$ and $0.83,0.87$ (each $3 \mathrm{H}, \mathrm{d}, J=5.9 \mathrm{~Hz}$, two methyls at C-2"), in addition to four singlet signals for four methyl groups at $\delta_{\mathrm{H}} 1.37,1.40,1.42$ and $1.54 .{ }^{13} \mathrm{C}$ and DEPT NMR spectra indicated the presence of eight methyls, two methylenes, four methines and twelve quaternary carbon atoms, three of the quaternary carbon atoms were assigned to three keto groups at $\delta_{\mathrm{C}}$ 212.12 (C-3), 206.48 (C-1') and 197.93 (C-1). The presence of isovaleryl and isobutyl groups was confirmed from ${ }^{13} \mathrm{C}$ signals at $\delta_{\mathrm{C}} 206.48,53.16,25.10,23.15$ and 22.80 and at $\delta_{\mathrm{C}} 45.83$, $25.12,23.48$ and 22.74, respectively. COSY data allowed the unambiguous assignment of two spin systems. The first spin system showed correlation of the methylene at $\delta_{\mathrm{H}} 2.99\left(\mathrm{H}_{2}-2^{\prime}\right)$, methine at $\delta_{\mathrm{H}} 2.28\left(\mathrm{H}-3^{\prime}\right)$ and two geminal methyls at $\delta_{\mathrm{H}} 0.98$ (2 methyls at C-3') corresponding to the isovaleryl group. The second consisted of a methine proton at $\delta_{\mathrm{H}} 4.26$ (H-9) correlated to a multiplet methylene signal at $\delta_{\mathrm{H}} 1.48\left(2 \mathrm{H}, \mathrm{m}, \mathrm{H}-1^{\prime \prime}\right)$, which further correlated with methine proton (overlapped with methyls) which gave a cross peak with two geminal methyl signals at $\delta_{\mathrm{H}}$ 0.83 and 0.87 , respectively. The assignment of protons and carbons was confirmed by HMQC data. The HMBC spectrum showed correlations of H-9 with C-1", 2", C-8a and 9a which established the connectivity of the isobutyl group at C-9. The position of the isovaleryl moiety at C-7 was confirmed by the HMBC correlation of $\mathrm{H}^{2}{ }^{\prime}$ with $\mathrm{C}-7$. The molecular weight, $\mathrm{UV}$, and NMR data of compound (3) were very similar to those reported for rhoydomyrtone ${ }^{10}$.

Eucalyptone G (4) was obtained as a yellow needle, physical characters, UV, ${ }^{1} \mathrm{H},{ }^{13} \mathrm{C}$ and DEPT NMR spectral data of 4 showed some similarities with those of 3 . IR spectrum showed absorption bands at $\gamma_{\max }(\mathrm{KBr}) \mathrm{cm}^{-1} ; 3390(\mathrm{OH}), 1715(\mathrm{C}=\mathrm{O})$ and $1584(\mathrm{C}=\mathrm{C})$. The ESI-MS showed a pseudomolecular ion peak at $m / z 677[\mathrm{M}+\mathrm{H}]^{+}(100 \%)$ which in conjunction with ${ }^{1} \mathrm{H}$, ${ }^{13} \mathrm{C}$ and DEPT NMR spectral data suggested the molecular formula $\mathrm{C}_{40} \mathrm{H}_{52} \mathrm{O}_{9}$. This was further confirmed by HRESI-MS. In comparison with $\mathbf{3}$, compound $\mathbf{4}$ required 15 degrees of unsaturation instead of 10 , which suggested the presence of an additional ring system. The significant differences in ${ }^{1} \mathrm{H}$ NMR between 3 and $\mathbf{4}$ were the appearance of new signals at $\delta_{\mathrm{H}}$ 
$4.39(1 \mathrm{H}, \mathrm{m})$, two methyls at $\delta_{\mathrm{H}} 0.85(6 \mathrm{H}, \mathrm{d}, J=6.0 \mathrm{~Hz})$ indicating the presence of isobutyryl group, four methyl signals, as well as the disappearance of the aromatic proton $\mathrm{H}-5$ in $4 .{ }^{13} \mathrm{C}$ and DEPT NMR showed the presence of additional six methyls, one methine, seven quaternary carbon atoms in (4), three of them were assigned to three keto groups at $\delta_{\mathrm{C}} 211.44\left(\mathrm{C}-3^{\prime \prime \prime}\right)$, $210.01\left(\mathrm{C}-7^{\prime \prime \prime}\right)$ and $197.47\left(\mathrm{C}-1^{\prime \prime \prime}\right)$ in addition to the lack the doublet ${ }^{13} \mathrm{C}$ NMR signal at $\delta_{\mathrm{C}}$ 94.76 (C-5). These data suggested that C-5 in (4) was blocked with a substituent. In COSY there is an additional spin system consisted of methine group and two methyl signals assigned to the isobutyryl moiety was observed. Comparison of the ${ }^{1} \mathrm{H}$ and ${ }^{13} \mathrm{C}$ NMR data with literature data ${ }^{10}$ indicated the presence of 5-(isobutyryl)-2,2,6,6-tetramethyl-cyclohex-4-ene-1,3-dione moiety [substructure A] (Figure 2) and was further confirmed by the presence of fragment ion peak at $m / z 441\left[\mathrm{M}^{-} \mathrm{C}_{14} \mathrm{H}_{19} \mathrm{O}_{3}\right]^{+}$in ESI-MS. The HMBC showed correlations of the 2 methyls at C-2"' with C-1" " and C-3"'; 2 methyls at C-4"' with C-3"' and C-5"'. The geminal dimethyls at $\delta_{\mathrm{H}}$ 0.82 correlated with $\mathrm{C}-7^{\prime \prime \prime}$; H-8"' with $\mathrm{C}-\mathrm{7}^{\prime \prime \prime}$ and $\mathrm{C}-5^{\prime \prime \prime}$ confirmed the attachment of the isobutyryl moiety at $\mathrm{C}-5^{\prime \prime \prime}$. The connectivity of the substructure $\mathbf{A}$ at $\mathrm{C}-5$ was established by the disappearance of $\mathrm{H}-5$ and downfield shift of C-5 to $\delta_{\mathrm{C}} 104.81$. To the best of our knowledge compound 4 is a new natural product and the name eucalyptone $\mathrm{G}$ was given to it. The known compounds were identified by analysis of the spectroscopic data (1D, 2D NMR and MS) and comparison of their data with those in the literature to be: stigmasterol $(\mathbf{1})^{11}$, ursolic acid (2) ${ }^{12}$, rhodomyrtone $(\mathbf{3})^{10}, 4^{\prime}, 5,7$-trimethoxy kaemepferol $(\mathbf{5})^{13,14}$, naringenin $(\mathbf{6})^{15}$, genistein $(7)^{13,14}$, catechin $(\mathbf{8})^{15,16}$, epicatechin $(\mathbf{9})^{15,16}$ and octyl- $\beta$-D-glucopyranoside $(\mathbf{1 0})^{17}$. This is the first report of compounds 1-3, 5, 7-10 in family Myrtaceae. Compound 6 was isolated for the first time from the plant.

Eucalyptone $\mathbf{G}$ was found to be active against the Gram-positive Bacillus subtilis and Staphylococcus aureus and caused an inhibition zone of $16 \mathrm{~mm}$ diameter after $24 \mathrm{~h}$ of incubation at $37{ }^{\circ} \mathrm{C}$. Also, highly active against Gram-negative E. coli with an inhibition zone of $19 \mathrm{~mm}$ diameter. 

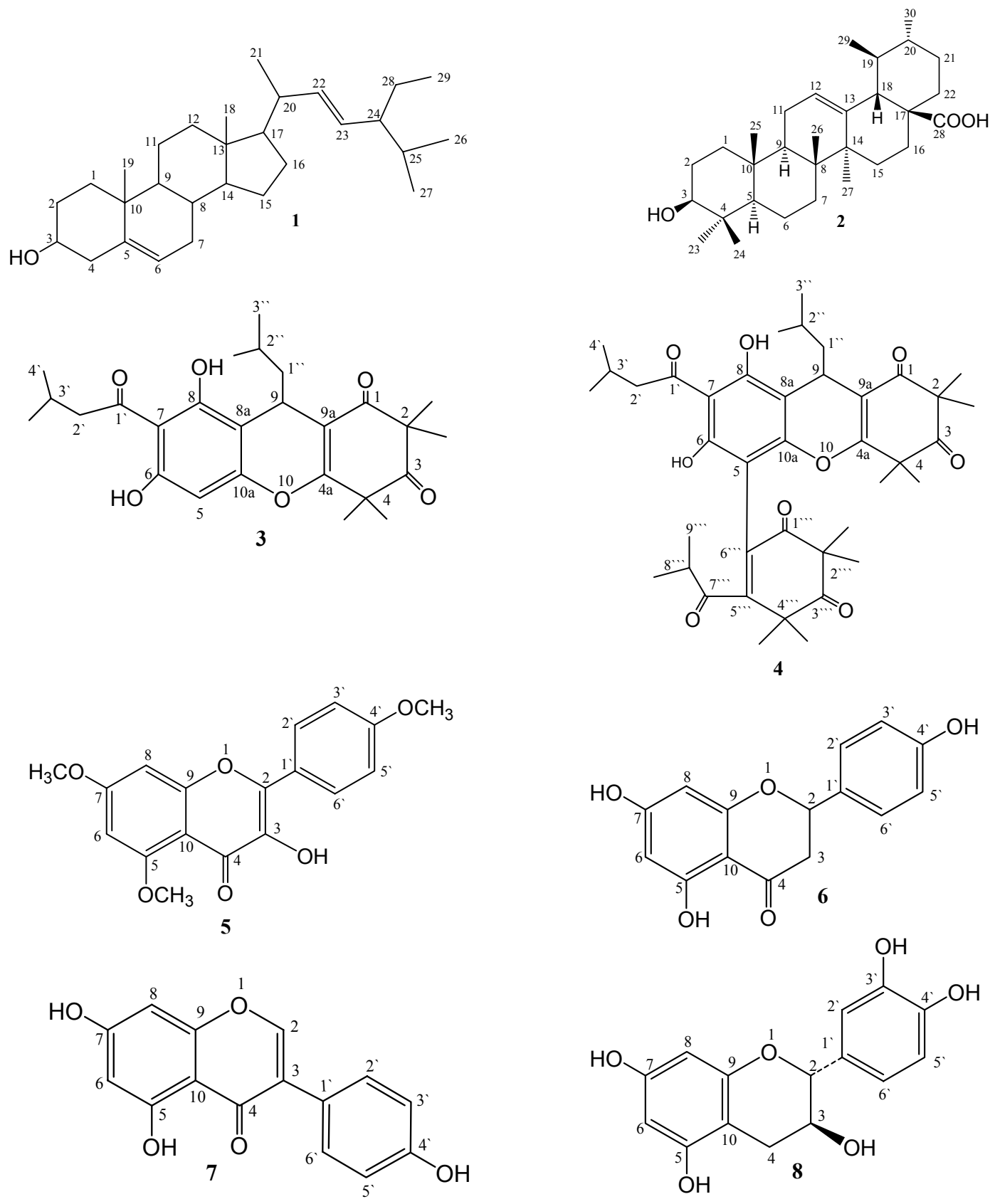<smiles>Oc1cc(O)c2c(c1)OC(c1ccc(O)c(O)c1)[C@H](O)C2</smiles><smiles>OC[C@H]1O[C@H](OCCCCCCCI)[C@@H](O)[C@H](O)[C@H]1O</smiles>

Figure 1. Chemical structures of the isolated compounds. 

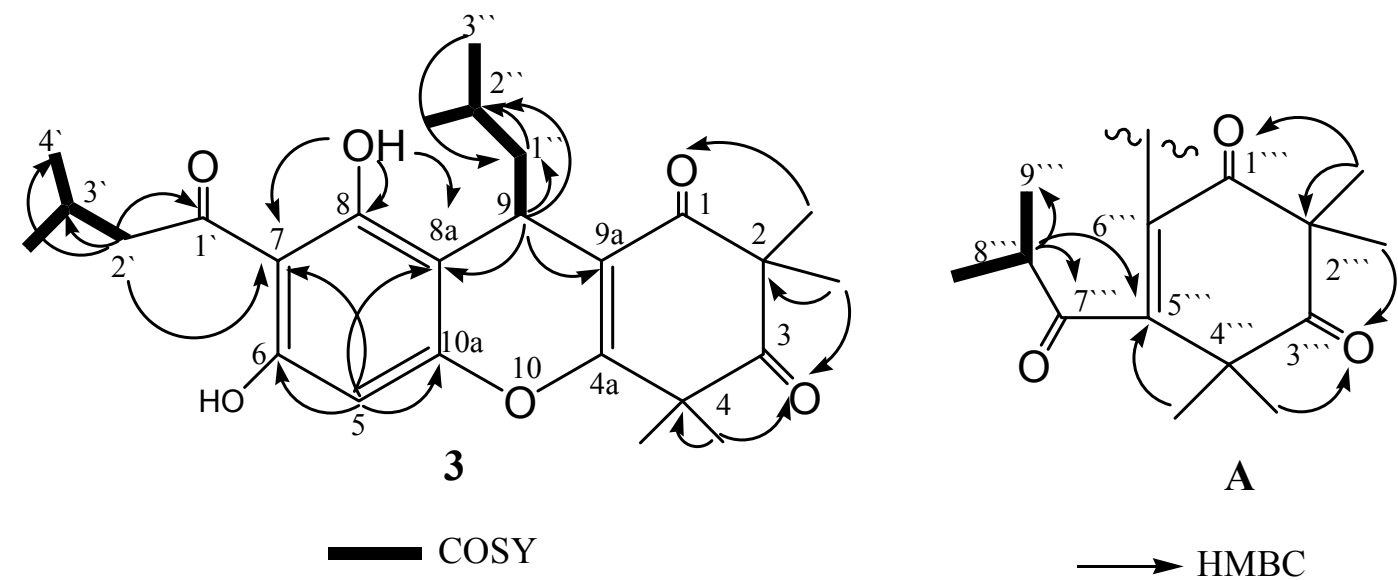

Figure 2. COSY and HMBC correlations of compound $\mathbf{3}$ and structure A.

\section{Experimental Section}

General Procedures. Melting points were determined in an Electrothermal 9100 Digital Melting Point (England). IR was measured on Schimadzu Infrared-400 spectrophotometer (Japan). The UV spectra were carried out in methanol (Merck) using a Perkin-Elmer Lambda 25 UV/VIS spectrophotometer. Electron impact mass spectra (EI-MS) were recorded on a Finnigan MAT TSQ 7000 mass spectrometer. Positive-ion electron spray ionization mass spectra (ESI-MS) were performed on a Thermofinnigan LCQ DECA mass spectrometer coupled to an Agilent 1100 HPLC system equipped with a photodiode-array detector. HRESI-MS was determined with a Micromass Qtof 2 mass spectrometer. ${ }^{1} \mathrm{H}$ and ${ }^{13} \mathrm{C}$ NMR spectra were measured on Bruker DRX 500 spectrometer (Bruker, Rheinstetten, Germany). 1D NMR spectral data were measured at temperature $23.7{ }^{\circ} \mathrm{C}$ in gradient sequences, while $2 \mathrm{D} \mathrm{NMR}$ at $26.85{ }^{\circ} \mathrm{C}$. High Performance Liquid Chromatography was performed on a HPLC system (Merck, Darmstadt, Germany) consisting of a Lachrom-Merck Hitachi L-7100 pump and an L-7400 UV detector using a C-18 column $(250 \times 8 \mathrm{~mm}$ i.d., prefilled with Eurospher 100, Knauer, Berlin, Germany) and UV detection at $\lambda 280 \mathrm{~nm}$. Vacuum liquid chromatography (VLC) was carried out on silica gel 60 (0.04-0.063 mm, $500 \mathrm{~g}$, Merck). Column chromatographic separations were performed over silica gel 60 (0.04-0.063 mm, Merck) and Sephadex LH-20 (0.25-0.1 mm Merck). TLC analyses were carried out on pre-coated silica gel $\mathrm{F}_{254}$ aluminium sheets and RP-18 $\mathrm{F}_{254 \mathrm{~s}}$ glass plates (Merck). Compounds were detected by UV absorption at $\lambda 255$ and $366 \mathrm{~nm}$ followed by spraying with anisaldehyde $/ \mathrm{H}_{2} \mathrm{SO}_{4}$ reagent and heating at $110{ }^{0} \mathrm{C}$ for $1-2 \mathrm{~min}$. The solvent systems used for TLC analyses were dichloromethane-methanol (97:3, solvent system I), dichloromethane-methanol (94:6, solvent system II) and dichloromethane-methanol (90:10, solvent system III). All solvents were distilled prior to use. 


\section{Extraction and isolation}

The bark of small twigs of Eucalyptus globulus Labill. was collected in August 2004 from trees growing on the margins of the ponds and banks of the Nile-river, Mankabad, Assiut, Egypt. A voucher specimen has been deposited at the Herbarium of Pharmacognosy Department, Faculty of Pharmacy, Al-Azhar University, Assiut branch, Assiut, Egypt.

The air-dried powdered bark $(900 \mathrm{~g})$ was extracted with methanol $(2.51 \times 4)$ at room temperature. The combined extract was concentrated under reduced pressure to afford a dark greenish brown residue $(46.0 \mathrm{~g})$. The latter was suspended in distilled water $(200 \mathrm{ml})$ then partitioned between $n$-hexane $(500 \mathrm{ml} \times 4)$, ethyl acetate $(500 \mathrm{ml} \times 4)$ and $n$-butanol $(500 \mathrm{ml} \times$ 3 ), successively. Each fraction was concentrated under reduced pressure to give $n$-hexane fraction (10.3 g), ethyl acetate fraction (7.6 g), $n$-butanol fraction (11 g) and aqueous (14.8 g) residue. The ethyl acetate fraction was subjected to VLC using $n$-hexane-ethyl acetate gradients, 6 fractions were obtained: E-1 to E-6. Fraction E-1 (1.1 g), fraction E-2 (0.89 g), fraction E-3 $(1.3 \mathrm{~g})$, fraction E-4 (0.95 g), fraction E-5 (1.4 g), and fraction E-6 (1.2 g). Fraction E-2 (0.89 g) was subjected to silica gel column chromatography $(120 \mathrm{~g} \times 50 \times 3 \mathrm{~cm})$ using dichloromethanemethanol 98:2 to afford compounds 1 (20 mg) and 2 (18 mg). Fraction E-3 (1.3 g) was subjected to VLC using dichloromethane-methanol gradients to obtain four subfractions E-3-A to E-3-D. Subfraction E-3-C (0.41 g) was subjected to silica gel column chromatography $(90.0 \mathrm{~g} \times 50 \times 3$ $\mathrm{cm}$ ) using dichloromethane-methanol $98: 2$ to afford compounds 3 (22 $\mathrm{mg}$ ) and 4 (9 $\mathrm{mg}$ ). Fraction E-4 (0.95 g) was chromatographed over Sephadex LH-20 column $(150 \mathrm{~g} \times 100 \times 5 \mathrm{~cm})$ using methanol-dichloromethane 9:1 as an eluent to obtain three main subfractions E-4-A to E-4C. Subfraction E-4-B (0.23 g) was subjected to silica gel column chromatography $(90.0 \mathrm{~g} \times 50 \times$ $3 \mathrm{~cm}$ ) using dichloromethane-methanol 92:8 to afford compounds 5 (14 mg), 6 (18 $\mathrm{mg})$ and 7 (23 mg). About $100 \mathrm{mg}$ of fraction E-5 was submitted to HPLC (HPLC gradient programm: 60:40 methanol/water at 0 and $5 \mathrm{~min} ; 100: 0 \mathrm{methanol} /$ water at 38 and $45 \mathrm{~min}$ and a flow rate 5.0 $\mathrm{ml} / \mathrm{min})$ to yield compound $8(19 \mathrm{mg})$ and $9(24 \mathrm{mg})$. Silica gel column chromatography of fraction E-6 (1.2 g) using dichloromethane-methanol 88:12 gave compound $\mathbf{1 0}(26 \mathrm{mg})$.

Stigmasterol (1). ${ }^{11}$ White needles; $\mathrm{R}_{f}=0.86$ (solvent system I); m. p. 169-170 ${ }^{\circ} \mathrm{C}$, EI-MS $\mathrm{m} / \mathrm{z}$ (rel. int.\%): $412[\mathrm{M}]^{+}(100) .{ }^{1} \mathrm{H} \mathrm{NMR}\left(500 \mathrm{MHz} \mathrm{CDCl}_{3}\right): \delta_{\mathrm{H}} 5.34(1 \mathrm{H}, \mathrm{dd}, J=2.8,5.1 \mathrm{~Hz}, \mathrm{H}-$ 6), $5.15(1 \mathrm{H}, \mathrm{dd}, J=8.8,15.1 \mathrm{~Hz}, \mathrm{H}-22), 5.01(1 \mathrm{H}, \mathrm{dd}, J=8.8,15.1 \mathrm{~Hz}, \mathrm{H}-23), 3.15(1 \mathrm{H}, \mathrm{m}, \mathrm{H}-$ 3), $1.02\left(3 \mathrm{H}, \mathrm{s}, \mathrm{CH}_{3}-19\right), 1.00\left(3 \mathrm{H}, \mathrm{d}, J=6.2 \mathrm{~Hz}, \mathrm{CH}_{3}-21\right), 0.84\left(3 \mathrm{H}, \mathrm{t}, J=6.3 \mathrm{~Hz}, \mathrm{CH}_{3}-29\right), 0.79$ $\left(3 \mathrm{H}, \mathrm{d}, J=7.5 \mathrm{~Hz}, \mathrm{CH}_{3}-26\right), 0.78\left(3 \mathrm{H}, \mathrm{d}, J=7.5 \mathrm{~Hz}, \mathrm{CH}_{3}-27\right), 0.69\left(3 \mathrm{H}, \mathrm{s}, \mathrm{CH}_{3}-18\right) .{ }^{13} \mathrm{C}$ NMR $\left(125 \mathrm{MHz}, \mathrm{CDCl}_{3}\right): \delta_{\mathrm{C}} 140.71(\mathrm{C}-5, \mathrm{~s}), 138.29$ (C-22, d), 129.24 (C-23, d), $121.69(\mathrm{C}-6, \mathrm{~d})$, 71.79 (C-3, d), 56.83 (C-17, d), 55.92 (C-14, d), 51.21 (C-24, d), 50.12 (C-9, d), 42.23 (C-13, s), 40.47 (C-20, d), 39.65 (C-4, t), 37.22 (C-1, t), 36.48 (C-10, s), 31.85 (C-8, 25, d), 31.59 (C-2, t), $31.50(\mathrm{C}-7, \mathrm{t}), 28.89$ (C-28, t), $25.38(\mathrm{C}-16, \mathrm{t}), 24.34$ (C-15, t), 21.19 (C-21, q), 21.07 (C-11, t), 21.05 (C-27, q), 19.37 (C-26, q), 18.96 (C-19, q), 12.23 (C-29, q), 12.02 (C-18, q).

Ursolic acid (2). ${ }^{12}$ White amorphous powder; $\mathrm{R}_{f}=0.71$ (solvent system I); m. p. $292-293{ }^{\circ} \mathrm{C}$, EI-MS $m / z$ (rel. int.\%): $456[\mathrm{M}]^{+}$(93). ${ }^{1} \mathrm{H}$ NMR (500 MHz, DMSO- $\left.d_{6}\right): \delta_{\mathrm{H}} 11.92$ (1H, s, 28$\mathrm{COOH}), 5.11(1 \mathrm{H}, \mathrm{dd}, J=3.8,6.9 \mathrm{~Hz}, \mathrm{H}-12), 4.28(1 \mathrm{H}, \mathrm{d}, J=15.0 \mathrm{~Hz}, 3-\mathrm{OH}), 2.98(1 \mathrm{H}, \mathrm{m}, \mathrm{H}-$ 
3), $2.10(1 \mathrm{H}, \mathrm{d}, J=11.0 \mathrm{~Hz}, \mathrm{H}-18), 1.02\left(3 \mathrm{H}, \mathrm{s}, \mathrm{CH}_{3}-23\right), 0.90\left(3 \mathrm{H}, \mathrm{d}, J=9.7 \mathrm{~Hz}, \mathrm{CH}_{3}-29\right), 0.88$ $\left(3 \mathrm{H}, \mathrm{s}, \mathrm{CH}_{3}-27\right), 0.85$ (3H, s, $\left.\mathrm{CH}_{3}-26\right), 0.80\left(3 \mathrm{H}, \mathrm{d}, J=6.6 \mathrm{~Hz}, \mathrm{CH}_{3}-30\right), 0.73\left(3 \mathrm{H}, \mathrm{s}, \mathrm{CH}_{3}-23\right)$, $0.66\left(3 \mathrm{H}, \mathrm{s}, \mathrm{CH}_{3}-25\right) .{ }^{13} \mathrm{C}$ NMR $\left(125 \mathrm{MHz}, \mathrm{DMSO}-d_{6}\right): \delta_{\mathrm{C}} 178.27(\mathrm{C}-28, \mathrm{~s}), 138.17(\mathrm{C}-13, \mathrm{~s})$, 124.55 (C-12, d), 76.81 (C-3, d), 54.76 (C-5, d), 52.35 (C-18, d), 47.00 (C-17, s), 46.80 (C-9, d), 41.62 (C-8, 14, s), 38.48 (C-4, s), 38.42 (C-19, d), 38.37 (C-20, d), 38.21 (C-1, t), 36.51 (C-22, t), $36.30(\mathrm{C}-10, \mathrm{~s}), 32.69(\mathrm{C}-7, \mathrm{t}), 30.17(\mathrm{C}-21, \mathrm{t}), 28.25(\mathrm{C}-23, \mathrm{q}), 27.53(\mathrm{C}-15, \mathrm{t}), 26.98(\mathrm{C}-2, \mathrm{t})$, 23.79 (C-16, t), 23.26 (C-27, q), 22.84 (C-11, t), 21.07 (C-30, q), 17.98 (C-6, t), 17.07 (C-26, q), 16.89 (C-29, q), 16.08 (C-25, q), 15.22 (C-24, q).

Rhodomyrtone (3). ${ }^{10}$ Yellow needles; $\mathrm{R}_{f}=0.78$ (solvent system I); m. p. $186-187{ }^{\circ} \mathrm{C}$, Positiveion ESI-MS $m / z$ (rel. int.\%): $443[\mathrm{M}+\mathrm{H}]^{+}(100)$, in addition to significant fragment peak at $\mathrm{m} / \mathrm{z}$ $385\left[\mathrm{M}-\mathrm{C}_{4} \mathrm{H}_{9}\right]^{+} .{ }^{1} \mathrm{H}$ NMR $\left(500 \mathrm{MHz}, \mathrm{CDCl}_{3}\right)$ see table $1,{ }^{13} \mathrm{C}$ NMR $\left(125 \mathrm{MHz}, \mathrm{CDCl}_{3}\right)$ : see table 2.

Table 1. ${ }^{1} \mathrm{H}$ NMR data of compounds 3 and $4\left(\mathrm{CDCl}_{3}, 500 \mathrm{MHz}\right)$

\begin{tabular}{|c|c|c|c|}
\hline \multicolumn{2}{|r|}{$3^{\mathrm{a}}$} & \multicolumn{2}{|r|}{$4^{\mathrm{a}}$} \\
\hline Pos. ${ }^{\mathrm{b}}$ & $\delta_{\mathrm{H}}(J \mathrm{~Hz}, \mathrm{~m})$ & Pos. ${ }^{b}$ & $\delta_{\mathrm{H}}(J \mathrm{~Hz}, \mathrm{~m})$ \\
\hline $2 \times \mathrm{CH}_{3}$ at pos. 2 & $1.37,1.40$, each $3 \mathrm{H}, \mathrm{s}$ & $\begin{array}{l}8 \times \mathrm{CH}_{3} \text { at pos. } \\
2,4,2^{\cdots}, 4^{\cdots}\end{array}$ & $1.35-1.64$, each $3 \mathrm{H}, \mathrm{s}$ \\
\hline $2 \times \mathrm{CH}_{3}$ at pos. 4 & $1.42,1.54$, each $3 \mathrm{H}, \mathrm{s}$ & & \\
\hline 5 & $6.11,1 \mathrm{H}, \mathrm{s}$ & 5 & - \\
\hline 9 & $4.26,1 \mathrm{H}, \mathrm{t}, J=5.4 \mathrm{~Hz}$ & 9 & $4.33,1 \mathrm{H}, \mathrm{t}, J=5.4 \mathrm{~Hz}$ \\
\hline 2 & $2.99,2 \mathrm{H}, \mathrm{dd}, J=6.8,15.5 \mathrm{~Hz}$ & 2 & $\begin{array}{c}\text { A } 3.21,1 \mathrm{H}, \mathrm{dd}, J= \\
6.3,17.3 \mathrm{~Hz} \\
\text { B } 3.00,1 \mathrm{H}, \mathrm{dd}, J= \\
\quad 6.3,17.3 \mathrm{~Hz}\end{array}$ \\
\hline $3^{\prime}$ & $2.28,1 \mathrm{H}, \mathrm{m}$ & 3 & $2.38,1 \mathrm{H}, \mathrm{m}$ \\
\hline $\begin{array}{c}2 \times \mathrm{CH}_{3} \text { at pos. } \\
3\end{array}$ & $0.98,6 \mathrm{H}, \mathrm{d}, J=6.6 \mathrm{~Hz}$ & $\begin{array}{c}2 \times \mathrm{CH}_{3} \text { at pos. } \\
3\end{array}$ & $1.03,6 \mathrm{H}, \mathrm{d}, J=6.3 \mathrm{~Hz}$ \\
\hline $1^{\prime \prime}$ & $1.48,2 \mathrm{H}, \mathrm{m}$ & $1^{\prime \prime}$ & overlapped with $\mathrm{CH}_{3}$ \\
\hline 2 & overlapped with $\mathrm{CH}_{3}$ & $2 "$ & overlapped with $\mathrm{CH}_{3}$ \\
\hline $\begin{array}{c}2 \times \mathrm{CH}_{3} \text { at pos. } \\
2^{\prime \prime}\end{array}$ & $\begin{array}{l}0.83,0.87, \text { each } 3 \mathrm{H}, \mathrm{d}, J=5.9 \\
\mathrm{~Hz}\end{array}$ & $\begin{array}{c}2 \times \mathrm{CH}_{3} \text { at pos. } \\
2^{\cdots} \\
8^{\cdots} \\
2 \times \mathrm{CH}_{3} \text { at pos. } \\
8^{\cdots}\end{array}$ & $\begin{array}{c}0.95,0.89, \text { each } 3 \mathrm{H}, \\
\mathrm{d}, J=5.9 \mathrm{~Hz} \\
4.39,1 \mathrm{H}, \mathrm{m} \\
0.82,6 \mathrm{H}, \mathrm{d} \\
J=6.0 \mathrm{~Hz}\end{array}$ \\
\hline $6-\mathrm{OH}$ & $7.59,1 \mathrm{H}, \mathrm{s}$ & 6-OH & - \\
\hline $8-\mathrm{OH}$ & $13.04,1 \mathrm{H}, \mathrm{s}$ & $8-\mathrm{OH}$ & $13.48,1 \mathrm{H}, \mathrm{s}$ \\
\hline
\end{tabular}

${ }^{\mathrm{a}}$ For clarity the same numbering system is used for $\mathbf{3}$ and $4{ }^{\mathrm{b}}$ Position. 
Table 2. ${ }^{13} \mathrm{C}$ NMR data of compounds 3 and $4\left(\mathrm{CDCl}_{3}, 125 \mathrm{MHz}\right)$

\begin{tabular}{|c|c|c|c|c|c|}
\hline \multicolumn{3}{|c|}{$3^{\mathrm{a}}$} & \multicolumn{3}{|c|}{$4^{\mathrm{a}}$} \\
\hline Pos. & $\delta_{\mathrm{C}}(\mathrm{m})$ & HMBC & Pos. & $\delta_{\mathrm{C}}(\mathrm{m})$ & HMBC \\
\hline 1 & $197.93 \mathrm{~s}$ & \multirow{5}{*}{1,2} & 1 & $197.47 \mathrm{~s}$ & \multirow{5}{*}{$1,2,1^{\cdots}, 2^{\cdots}$} \\
\hline 2 & $56.05 \mathrm{~s}$ & & 2 & $56.28 \mathrm{~s}$ & \\
\hline $\begin{array}{l}2 \times \mathrm{CH}_{3} \\
\text { at pos. } 2\end{array}$ & $\begin{array}{l}24.56 \mathrm{q}, \\
24.58 \mathrm{q}\end{array}$ & & $\begin{array}{c}4 \times \mathrm{CH}_{3} \\
\text { at pos. } 2,2 \cdots\end{array}$ & $\begin{array}{l}23.82 \mathrm{q}, 24.19 \mathrm{q} \\
25.04 \mathrm{q}, 25.56 \mathrm{q}\end{array}$ & \\
\hline 3 & $212.12 \mathrm{~s}$ & & 3 & $211.67 \mathrm{~s}$ & \\
\hline 4 & $47.15 \mathrm{~s}$ & & 4 & $47.25 \mathrm{~s}$ & \\
\hline $2 \times \mathrm{CH}_{3}$ & $24.71 \mathrm{q}$, & \multirow[t]{3}{*}{$4,4 a$} & $4 \times \mathrm{CH}_{3}$ & $24.36 \mathrm{q}, 24.68 \mathrm{q}$, & \multirow{8}{*}{$\begin{array}{c}4,4 \mathrm{a} \\
4^{\cdots}, 5^{\cdots}\end{array}$} \\
\hline at pos. 4 & $24.14 \mathrm{q}$ & & at pos. $4,4^{\prime \prime}$ & $24.87 \mathrm{q}, 24.94 \mathrm{q}$ & \\
\hline $4 a$ & $167.14 \mathrm{~s}$ & & $4 a$ & $166.76 \mathrm{~s}$ & \\
\hline 5 & $94.76 \mathrm{~d}$ & \multirow[t]{5}{*}{$6,7,8 \mathrm{a}, 10 \mathrm{a}$} & 5 & $104.81 \mathrm{~s}$ & \\
\hline 6 & $158.15 \mathrm{~s}$ & & 6 & $152.35 \mathrm{~s}$ & \\
\hline 7 & $107.58 \mathrm{~s}$ & & 7 & $107.58 \mathrm{~s}$ & \\
\hline 8 & $162.61 \mathrm{~s}$ & & 8 & $160.61 \mathrm{~s}$ & \\
\hline $8 \mathrm{a}$ & $106.49 \mathrm{~s}$ & & $8 \mathrm{a}$ & $105.67 \mathrm{~s}$ & \\
\hline 9 & $25.15 \mathrm{~d}$ & \multirow[t]{4}{*}{$8 \mathrm{a}, 9 \mathrm{a}, 1^{\prime \prime}, 2^{\prime \prime}$} & 9 & $25.26 \mathrm{~d}$ & \multirow[t]{4}{*}{$8 \mathrm{a}, 9 \mathrm{a}, 1^{\prime \prime}, 2^{\prime \prime}$} \\
\hline $9 \mathrm{a}$ & $114.02 \mathrm{~s}$ & & $9 \mathrm{a}$ & $114.27 \mathrm{~s}$ & \\
\hline $10 \mathrm{a}$ & $155.61 \mathrm{~s}$ & & $10 \mathrm{a}$ & $154.91 \mathrm{~s}$ & \\
\hline 1 & $206.48 \mathrm{~s}$ & & 1 & $204.58 \mathrm{~s}$ & \\
\hline 2 & $53.16 \mathrm{t}$ & \multirow[t]{2}{*}{$1,3 \nmid 7$} & 2 & $53.90 \mathrm{t}$ & \multirow[t]{2}{*}{$1,3,7$} \\
\hline 3 & $25.10 \mathrm{~d}$ & & 3 & $25.11 \mathrm{~d}$ & \\
\hline $2 \times \mathrm{CH}_{3}$ & $23.15 \mathrm{q}$, & \multirow[t]{2}{*}{$1^{\prime}, 2^{\prime}, 3^{\prime}$} & $2 \times \mathrm{CH}_{3}$ & $23.27 \mathrm{q}$, & \multirow[t]{2}{*}{$1^{\prime}, 2^{\prime}, 3^{\prime}$} \\
\hline at pos. $3^{\prime}$ & $22.80 \mathrm{q}$ & & at pos. $3^{\prime}$ & $23.30 \mathrm{q}$ & \\
\hline $1^{\prime \prime}$ & $45.83 \mathrm{t}$ & \multirow[t]{2}{*}{$9,2^{\prime \prime}$} & $1 "$ & $45.47 \mathrm{t}$ & \multirow[t]{2}{*}{$9,2^{\prime \prime}$} \\
\hline $2 "$ & $25.12 \mathrm{~d}$ & & $2^{\prime \prime}$ & $25.26 \mathrm{~d}$ & \\
\hline \multirow{12}{*}{$\begin{array}{c}2 \times \mathrm{CH}_{3} \\
\text { at pos. } 2^{\prime}\end{array}$} & $22.74 \mathrm{q}$, & \multirow[t]{12}{*}{$1^{\prime \prime}, 2^{\prime \prime}$} & $2 \times \mathrm{CH}_{3}$ & $23.35 \mathrm{q}$ & \multirow[t]{9}{*}{$1^{\prime \prime}, 2^{\prime \prime}$} \\
\hline & $23.48 \mathrm{q}$ & & at pos. $2^{\prime \prime}$ & $23.78 \mathrm{q}$ & \\
\hline & & & $1 \cdots$ & $197.44 \mathrm{~s}$ & \\
\hline & & & $2^{\cdots}$ & $56.03 \mathrm{~s}$ & \\
\hline & & & $3^{\cdots \cdots}$ & $211.44 \mathrm{~s}$ & \\
\hline & & & $4^{\prime \prime}$ & $47.28 \mathrm{~s}$ & \\
\hline & & & $5^{\prime \prime}$ & $113.52 \mathrm{~s}$ & \\
\hline & & & $6^{\prime \prime}$ & $111.74 \mathrm{~s}$ & \\
\hline & & & $7^{\cdots}$ & $210.01 \mathrm{~s}$ & \\
\hline & & & $8^{\cdots}$ & $39.21 \mathrm{~d}$ & $\begin{array}{c}5^{\cdots,}, 7^{\cdots}, 2 \times \\
\mathrm{CH}_{3} \\
\text { at } 8^{\cdots}\end{array}$ \\
\hline & & & $2 \times \mathrm{CH}_{3}$ & $22.82 \mathrm{q}$ & $7^{\cdots}, 8^{\cdots}$ \\
\hline & & & $\begin{array}{c}\text { at pos. } 8^{\prime \prime} \\
8-\mathrm{OH}\end{array}$ & $22.64 \mathrm{q}$ & $7,8,8 \mathrm{a}$ \\
\hline
\end{tabular}

${ }^{a}$ For clarity the same numbering system is used for $\mathbf{3}$ and $\mathbf{4}$. 
Eucalyptone G (4). Yellow needles; $\mathrm{R}_{f}=0.68$ (solvent system I); m. p. 197-198 ${ }^{\circ} \mathrm{C}$, Positive-ion ESI-MS $m / z$ (rel. int.\%): $677[\mathrm{M}+\mathrm{H}]^{+}$(88); HRESI-MS $m / z 677.3647$ (calcd for $\mathrm{C}_{40} \mathrm{H}_{52} \mathrm{O}_{9}$ 677.3611) in addition to a significant fragment ion peak at $m / z 441$ [M-236] .IR $\gamma_{\max }(\mathrm{KBr}) \mathrm{cm}^{-1}$ : 3390, 2975, 1715, 1709, 1620, 1584, 1460, 1323, 1120, 762. ${ }^{1} \mathrm{H}$ NMR (500 MHz, $\mathrm{CDCl}_{3}$ ): see table $1,{ }^{13} \mathrm{C}$ NMR $\left(125 \mathrm{MHz}, \mathrm{CDCl}_{3}\right)$ : see table 2 .

4,5,7-Trimethoxykaempferol (5). ${ }^{13,14}$ Yellow needles; $\mathrm{R}_{f}=0.54$ (solvent system I); m. p. 152 ${ }^{\circ} \mathrm{C}$, Positive-ion ESI-MS m/z (rel. int.\%): $329[\mathrm{M}+\mathrm{H}]{ }^{+}(100) .{ }^{1} \mathrm{H}$ NMR $\left(500 \mathrm{MHz}, \mathrm{CDCl}_{3}\right): \delta_{\mathrm{H}}$ $8.19\left(2 \mathrm{H}, \mathrm{dd}, J=1.9,6.9 \mathrm{~Hz}, \mathrm{H}-2^{`}, 6^{`}\right), 7.05\left(2 \mathrm{H}, \mathrm{dd}, J=1.9,6.9 \mathrm{~Hz}, \mathrm{H}-3^{`}, 5^{`}\right), 6.57$ (1H, d, $J=$ $2.2 \mathrm{~Hz}, \mathrm{H}-8), 6.36(1 \mathrm{H}, \mathrm{d}, J=2.2 \mathrm{~Hz}, \mathrm{H}-6), 3.88\left(3 \mathrm{H}, \mathrm{s}, 5-\mathrm{OCH}_{3}\right), 3.91\left(3 \mathrm{H}, \mathrm{s}, 7-\mathrm{OCH}_{3}\right), 3.98$ $\left(3 \mathrm{H}, \mathrm{s}, 4{ }^{-}-\mathrm{OCH}_{3}\right) .{ }^{13} \mathrm{C}$ NMR $\left(125 \mathrm{MHz}, \mathrm{CDCl}_{3}\right): \delta_{\mathrm{C}} 171.93(\mathrm{C}-4, \mathrm{~s}), 164.29(\mathrm{C}-7, \mathrm{~s}), 160.61(\mathrm{C}-$ 5, s), 160.53 (C-4', s), 158.85 (C-2, s), 142.25 (C-9, s), 137.43 (C-3, s), 128.86 (C-2`, 6’, d), 123.56 (C-1 ', s), 113.99 (C-3', 5’, d), 106.24 (C-10, s), 95.64 (C-6, d), 92.39 (C-8, d), 56.39 (4` $\left.\mathrm{OCH}_{3}, \mathrm{q}\right), 55.79\left(7-\mathrm{OCH}_{3}, \mathrm{q}\right), 55.37\left(5-\mathrm{OCH}_{3}, \mathrm{q}\right)$.

Naringenin (6). ${ }^{15}$ Yellowish white amorphous powder; $\mathrm{R}_{f}=0.86$ (solvent system II); m. p. 257$258{ }^{\circ} \mathrm{C}$, Positive-ion ESI-MS $\mathrm{m} / z$ (rel. int.\%): $273[\mathrm{M}+\mathrm{H}]^{+}$(100). ${ }^{1} \mathrm{H}$ NMR (500 MHz, DMSO$\left.d_{6}\right): \delta_{\mathrm{H}} 12.14(1 \mathrm{H}, \mathrm{s}, 5-\mathrm{OH}), 10.77(1 \mathrm{H}, \mathrm{s}, 7-\mathrm{OH}), 9.57(1 \mathrm{H}, \mathrm{s}, 4-\mathrm{OH}), 7.30(2 \mathrm{H}, \mathrm{d}, J=8.5 \mathrm{~Hz}$, H-2`, 6`), 6.78 (2H, d, $J=8.5$ Hz, H-3`, 5`), 5.86 (2H, br s, H-6, 8), $5.42(1 \mathrm{H}, \mathrm{dd}, J=2.8,12.6$ $\mathrm{Hz}, \mathrm{H}-2), 3.26\left(1 \mathrm{H}, \mathrm{dd}, J=12.9,17.0 \mathrm{~Hz}, \mathrm{H}-3_{\mathrm{ax}}\right), 2.67\left(1 \mathrm{H}, \mathrm{dd}, J=2.8,17.0 \mathrm{~Hz}, \mathrm{H}-3_{\mathrm{eq}}\right) .{ }^{13} \mathrm{C}$ NMR (125 MHz, DMSO-d $d_{6}$ ): $\delta_{\mathrm{C}} 196.40$ (C-4, s), 166.62 (C-7, s), 163.47 (C-5, s), 162.93 (C-9,

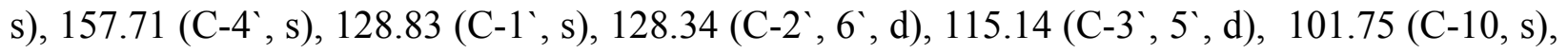
95.76 (C-6, d), 94.94 (C-8, d), 78.41 (C-2, d), 41.95 (C-3, t).

Genistein (7). ${ }^{13,14}$ Yellow amorphous powder; $\mathrm{R}_{f}=0.77$ (solvent system II); m. p. 303-304 ${ }^{\circ} \mathrm{C}$, Positive-ion ESI-MS $m / z$ (rel. int.\%): $271[\mathrm{M}+\mathrm{H}]^{+}(100) .{ }^{1} \mathrm{H}$ NMR (500 MHz, DMSO- $\left.d_{6}\right): \delta_{\mathrm{H}}$ $12.94(1 \mathrm{H}, \mathrm{s}, 5-\mathrm{OH}), 10.87(1 \mathrm{H}, \mathrm{s}, 7-\mathrm{OH}), 9.58\left(1 \mathrm{H}, \mathrm{s}, 4^{-}-\mathrm{OH}\right), 8.31$ (1H, s, H-2), 7.30 (2H, dd, $J$ $\left.=1.9,6.6 \mathrm{~Hz}, \mathrm{H}-2^{`}, 6^{`}\right), 6.78\left(2 \mathrm{H}, \mathrm{dd}, J=1.9,6.6 \mathrm{~Hz}, \mathrm{H}-3^{`}, 5^{`}\right), 6.37$ (1H, d, $\left.J=2.2 \mathrm{~Hz}, \mathrm{H}-8\right)$, $6.21(1 \mathrm{H}, \mathrm{d}, J=2.2 \mathrm{~Hz}, \mathrm{H}-6) .{ }^{13} \mathrm{C}$ NMR (125 MHz, DMSO- $\left.d_{6}\right): \delta_{\mathrm{C}} 180.20(\mathrm{C}-4, \mathrm{~s}), 164.25(\mathrm{C}-7$, s), 161.98 (C-5, s), 157.57 (C-4', s), 157.40 (C-9, s), 153.98 (C-2, d), 130.15 (C-2', 6', d), 122.25 (C-3, s), 121.18 (C-1', s), 115.04 (C-3`, 5', d), 104.45 (C-10, s), 98.95 (C-6, d), 93.64 (C-8, d).

Catechin (8). ${ }^{15,16}$ Whitish powder; $\mathrm{R}_{f}=0.69$ (solvent system II); m. p. 244-245 ${ }^{\circ} \mathrm{C}$, Positive-ion ESI-MS $m / z$ (rel. int.\%): $291[\mathrm{M}+\mathrm{H}]^{+}(100) .{ }^{1} \mathrm{H}$ NMR (500 MHz, DMSO- $\left.d_{6}\right): \delta_{\mathrm{H}} 9.17$ (1H, s, 5$\mathrm{OH}), 8.93(1 \mathrm{H}, \mathrm{s}, 7-\mathrm{OH}), 8.86\left(1 \mathrm{H}, \mathrm{s}, 4{ }^{`}-\mathrm{OH}\right), 8.81\left(1 \mathrm{H}, \mathrm{s}, 3{ }^{`}-\mathrm{OH}\right), 6.70(1 \mathrm{H}, \mathrm{d}, J=1.6 \mathrm{~Hz}, \mathrm{H}-$ $\left.2^{`}\right), 6.67(1 \mathrm{H}, \mathrm{d}, J=8.2 \mathrm{~Hz}, \mathrm{H}-6$ '), 6.57 (1H, dd, $J=1.6,8.2 \mathrm{~Hz}, \mathrm{H}-5$ '), 5.87 (1H, d, $J=2.2 \mathrm{~Hz}$, $\mathrm{H}-8), 5.67(1 \mathrm{H}, \mathrm{d}, J=2.2 \mathrm{~Hz}, \mathrm{H}-6), 4.86(1 \mathrm{H}, \mathrm{d}, J=5.86 \mathrm{~Hz}, \mathrm{H}-2), 4.47(1 \mathrm{H}, \mathrm{d}, J=7.6 \mathrm{~Hz}, 3-$ $\mathrm{OH}), 3.80(1 \mathrm{H}, \mathrm{ddd}, J=5.3,7.5,12.9 \mathrm{~Hz}, \mathrm{H}-3), 2.64(1 \mathrm{H}, \mathrm{dd}, J=5.3,16.1 \mathrm{~Hz}, \mathrm{H}-4$ eq $), 2.33(1 \mathrm{H}$, $\mathrm{dd}, J=7.9,15.7 \mathrm{~Hz}, \mathrm{H}-4 \mathrm{ax}) .{ }^{13} \mathrm{C}$ NMR $\left(125 \mathrm{MHz}, \mathrm{DMSO}-d_{6}\right): \delta_{\mathrm{C}} 156.45$ (C-5, s), 156.17 (C-7, s), 155.35 (C-9, s), 144.83 (C-3`, 4’, s), 130.58 (C-1', s), 118.34 (C-6`, d), 115.06 (C-5`, d), 114.15 (C-2', d), 99.05 (C-10, s), 95.09 (C-6, d), 93.83 (C-8, d), 80.99 (C-2, d), 66.30 (C-3, d), $27.87(\mathrm{C}-4, \mathrm{t})$.

Epicatechin (9). ${ }^{15,16}$ Yellowish residue; $\mathrm{R}_{f}=0.69$ (solvent system II); m. p. 241-242 ${ }^{\circ} \mathrm{C}$, Positive-ion ESI-MS m/z (rel. int.\%): $291[\mathrm{M}+\mathrm{H}]^{+}$(100). ${ }^{1} \mathrm{H}$ NMR (500 MHz, DMSO- $d_{6}$ ): $\delta_{\mathrm{H}}$ 
$9.17(1 \mathrm{H}, \mathrm{s}, 5-\mathrm{OH}), 8.93(1 \mathrm{H}, \mathrm{s}, 7-\mathrm{OH}), 8.85\left(1 \mathrm{H}, \mathrm{s}, 4^{`}-\mathrm{OH}\right), 8.75\left(1 \mathrm{H}, \mathrm{s}, 3^{`}-\mathrm{OH}\right), 6.96(1 \mathrm{H}, \mathrm{d}, J$ $\left.=1.6 \mathrm{~Hz}, \mathrm{H}-2^{`}\right), 6.75\left(1 \mathrm{H}, \mathrm{d}, J=8.2 \mathrm{~Hz}, \mathrm{H}-6{ }^{`}\right), 6.71\left(1 \mathrm{H}, \mathrm{dd}, J=1.6,8.2 \mathrm{~Hz}, \mathrm{H}-5^{`}\right), 5.94(1 \mathrm{H}, \mathrm{d}$, $J=2.2 \mathrm{~Hz}, \mathrm{H}-8), 5.75(1 \mathrm{H}, \mathrm{d}, J=2.2 \mathrm{~Hz}, \mathrm{H}-6), 4.82(1 \mathrm{H}, \mathrm{s}, \mathrm{H}-2), 4.69$ (1H, d, $J=5.42 \mathrm{~Hz}, 3-$ $\mathrm{OH}), 4.07$ (1H, d, $J=3.4 \mathrm{~Hz}, \mathrm{H}-3), 2.64\left(1 \mathrm{H}, \mathrm{dd}, J=4.1,16.1 \mathrm{~Hz}, \mathrm{H}-4_{\mathrm{ax}}\right), 2.33(1 \mathrm{H}, \mathrm{dd}, J=2.2$, $\left.15.4 \mathrm{~Hz}, \mathrm{H}-4_{\text {eq }}\right) .{ }^{13} \mathrm{C}$ NMR $\left(125 \mathrm{MHz}, \mathrm{DMSO}-d_{6}\right): \delta_{\mathrm{C}} 156.79(\mathrm{C}-5, \mathrm{~s}), 156.47$ (C-7, s), 156.03 (C9, s), 144.73 (C- 4', s), 144.66 (C-3`, s), 130.90 (C-1`, s), 118.27 (C-6’ d), 115.12 (C-2`, d), 115.06 (C-5 , d), 98.79 (C-10, s), 95.36 (C-6, d), 94.38 (C-8, d), 78.31 (C-2, d), 65.19 (C-3, d), $28.47(\mathrm{C}-4, \mathrm{t})$.

Octyl- $\beta$-D-glucopyranoside (10). ${ }^{17}$ White powder; $\mathrm{R}_{f}=0.62$ (solvent system III); m. p. $107{ }^{\circ} \mathrm{C}$, FAB-MS $m / z$ (rel. int.\%): $315[\mathrm{M}+\mathrm{Na}]^{+}(100) .{ }^{1} \mathrm{H}$ NMR (500 MHz, DMSO- $\left.d_{6}\right): \delta_{\mathrm{H}} 4.92(1 \mathrm{H}, \mathrm{s}$, $\left.2^{\circ}-\mathrm{OH}\right), 4.89\left(1 \mathrm{H}, \mathrm{s}, 3^{`}\right.$ or $\left.4^{`}-\mathrm{OH}\right), 4.86\left(1 \mathrm{H}, \mathrm{s}, 5^{`}-\mathrm{OH}\right), 4.08\left(1 \mathrm{H}, \mathrm{d}, J=7.9 \mathrm{~Hz}, \mathrm{H}-1^{`}\right), 3.72$, 3.41 (2H, m, H-6 ) , 3.72 (1H, m, H-8A), 3.40 (1H, m, H-8B), 3.10 (1H, m, H-5 '), 3.06-3.03 (2H, m, H-3`, 4'), 2.90 (1H, m, H-2`), 1.49 (2H, quintet, $J=7.2 \mathrm{~Hz}, \mathrm{H}-7), 1.29-1.24$ (12 H, br s, $\left.\left(\mathrm{CH}_{2}\right)_{6^{-}}\right), 0.84\left(3 \mathrm{H}, \mathrm{d}, J=7.2 \mathrm{~Hz}, \mathrm{H}_{3}-1\right) .{ }^{13} \mathrm{C}$ NMR $\left(125 \mathrm{MHz}, \mathrm{DMSO}-d_{6}\right): \delta_{\mathrm{C}} 102.81\left(\mathrm{C}-1^{`}, \mathrm{~d}\right)$, 76.79 (C-3`, d), 76.75 (C-5`, d), 73.41 (C-2`, d), 70.05 (C-4`, d), 68.52 (C-8, t), 61.06 (C-6’, t), 31.24 (C-7, t), 29.29 (C-6, t), 28.88 (C-5, t), 28.68 (C-4, t), $25.52(\mathrm{C}-3, \mathrm{t}), 22.02(\mathrm{C}-2, \mathrm{t}), 13.95$ $(\mathrm{C}-1, \mathrm{q})$.

Agar plate diffusion assay. ${ }^{18}$ Susceptibility disks $(5 \mathrm{~mm}$ diameter) were impregnated with $5 \mu \mathrm{g}$ of the isolated compound and then placed on agar plates inoculated with the test bacterium: $B$. subtilis 168 and S. aureus ATCC 25923 (for the Gram-positive bacteria) and E. coli ATCC 25922 (for Gram-negative bacteria). The plates were observed for zones of inhibition, after $24 \mathrm{~h}$ of incubation at $37{ }^{\circ} \mathrm{C}$. In all cases, for the controls containing only the respective amount of solvent, no zones of inhibition were observed.

\section{Acknowledgements}

The authors are grateful to Professor Peter Proksch Institut für Pharmazeutische Biologie, Heinrich-Heine-Universität Düsseldorf, Universitätsstraße 1, Geb. 26.23, D-40225 Düsseldorf, Germany for carrying HRESI-MS measurement and antibacterial activity testing.

\section{References}

1. Ghisalberti, E. L. Phytochemistry 1996, 41, 7.

2. 2. Ito, H.; Koreshi, M.; Tokuda, H.; Nishino, H.; Yashida, T. J. Nat. Prod. 2000, 63, 1253.

3. Kozuka, M.; Sawada, T.; Kasahara, F.; Mizuta, E.; Amano, T.; Komiya, T.; Goto, M. Chem. Pharm. Bull. 1982, 30, 1952.

4. Kozuka, M.; Sawada, T.; Mizuta, E.; Kasahara, F.; Amano, T.; Komiya, T.; Goto, M. Chem. Pharm. Bull. 1982, 30, 1964. 
5. Noble, H. M.; Sidebottom, P. J.; Lane, S. J.; O’Neill, M. J. Planta Med. 1990, 56, 647.

6. Osawa, K.; Yasuda, H.; Morita, H.; Takeya, K.; Itokawa, H. Phytochemistry 1995, 40, 183.

7. Osawa, K.; Yasuda, H.; Morita, H.; Takeya, K.; Itokawa, H. J. Nat. Prod. 1996, 59, 823.

8. Courtney, J. L.; Lassak, E. V.; Speirs, G. B. Phytochemistry 1983, 22, 947.

9. Umehara, K.; Singh, I. P.; Etoh, H.; Takasaki, M.; Konoshima, T. Phytochemistry 1998, 49, 1699.

10. Dachriyanus, Salni, Sargent, M. V.; Skelton, B. W.; Soediro, I.; Sutisna, M.; White, A. L.; Yulinah, E. Aust. J. Chem. 2002, 55, 229.

11. Asakawa, Y.; Toyota, M.; Harrison, L. J. Phytochemistry 1985, 24, 1505.

12. Seebacher, W.; Simic, N.; Weis, R.; Saf, R.; Kunert, O. Magn. Reson. Chem. 2003, 41, 636.

13. Mabry, T. J.; Markham, K. R.; Thomas, M. B. The Systematic Identification of Flavonoids; Springer Verlag: New York, Heidelberg, Berlin, 1970.

14. Harborne, J. B. The Flavonoids Advances in Research Since 1986; Chapman and Hall: London, 1994.

15. Shen, C. C.; Chang, Y. S.; Ho, L. K. Phytochemistry 1993, 34, 843.

16. Rossouw, W.; Hundt, M. F.; Steenkamp, J. A.; Ferreira, D. Tetrahedron 1994, 50, 12477.

17. Fan, W.; Tezuka, Y.; Ni, K. M.; Kadota, S. Chem. Pharm. Bull. 2001, 49, 396.

18. Edrada, R. A.; Proksch, P.; Wray, V.; Witte, L.; Müller, W. E. G.; Van Soest, R. W. M. J. Nat. Prod. 1996, 59, 1056. 